\title{
Pour une école inclusive : des intentions aux réalisations
}

Introduction

Introduction. For an inclusive school: From intentions to achievements

Introducción. Por una escuela inclusiva: de las intenciones a las realizaciones

\section{Abdeljalil Akkari et Valérie Barry}

\section{CpenEdition}

Journals

Édition électronique

URL : https://journals.openedition.org/ries/6394

DOI : 10.4000/ries.6394

ISSN : 2261-4265

Éditeur

France Education international

Édition imprimée

Date de publication : 1 septembre 2018

Pagination : 37-46

ISBN : 978-2-85420-619-7

ISSN : $1254-4590$

Référence électronique

Abdeljalil Akkari et Valérie Barry, "Pour une école inclusive : des intentions aux réalisations 》, Revue internationale d'éducation de Sèvres [En ligne], 78 I septembre 2018, mis en ligne le 01 septembre 2018, consulté le 24 juin 2021. URL : http://journals.openedition.org/ries/6394 ; DOI : https://doi.org/ $10.4000 /$ ries.6394 


\title{
Accueillir tous les enfants à l'école : la question de l'inclusion
}

\author{
Introduction \\ Pour une école inclusive : des intentions aux réalisations
}

\author{
Abdeljalil Akkari \\ Université de Genève \\ Valérie Barry \\ ESPE de l'Université Paris-Est Créteil
}

\section{L'ENJEU MONDIAL}

\section{ET LES PRINCIPES D'UNE ÉCOLE} DE LA DIVERSITÉ

Quel que soit le terme utilisé (inclusion, éducation inclusive, école inclusive, etc.), le discours contemporain sur l'éducation place la question de l'inclusion au cœur des problématiques éducatives. La notion d'inclusion a été réaffirmée au plan international lors de la Conférence mondiale sur l'éducation d'Incheon et lors de l'adoption de l'Agenda 2030 par les Nations Unies, en septembre 2015. Alors qu'à l'origine, la problématique de l'inclusion se focalisait sur l'intégration scolaire des apprenants en situation de handicap ou présentant des troubles de l'apprentissage, elle a progressivement été élargie pour englober les besoins des élèves migrants, des minorités ethniques ou genrées et d'autres catégories socioculturelles ne bénéficiant pas d'un accès équitable à l'éducation et à la formation. Désormais, l'école inclusive veille dans ses objectifs à l'inclusion scolaire de tous les enfants, sans aucune distinction, offre les mêmes chances à tous et prend en compte la diversité des élèves.

Plus largement, l'école inclusive est l'échelle micro d'une société inclusive, d'un investissement global de tous pour chacun. En Europe, les indicateurs structurels de l'inclusion sont soutenus par dix principes clés, qui englobent notamment :

- une attention portée de façon systémique au traitement des blocages ;

- en articulation, une approche holistique, qui reconnaît les besoins sociaux, émotionnels et instrumentaux des élèves, et non seulement leurs besoins scolaires et cognitifs ;

- un principe d'égalité et de non-discrimination, qui reconnaît que différents groupes peuvent avoir besoin de soutiens supplémentaires dans un environnement respectueux, dépourvu de préjugés ; 
- un principe d'écoute de la voix des enfants, qui requiert un engagement touchant directement au bien-être de ceux-ci et prenant en compte leur âge et leur maturité ;

- un principe de construction basé sur les atouts et les potentialités des personnes, qui remet en question les étiquettes négatives associées aux groupes vulnérables, et qui se situe au-delà d'une simple prévention de difficultés, en cherchant à promouvoir chacun ;

- un principe de participation active des parents à l'école, qui requiert une orientation stratégique touchant aux parents marginalisés;

- un principe de différentiation, qui pointe le fait que des niveaux de besoin différents requièrent des stratégies de prévention différentes;

- un principe de multidisciplinarité, qui reconnaît la nécessité de réponses multiformes face à des besoins complexes;

- un principe de représentation des groupes historiquement marginalisés, qui implique une attention aux processus favorisant leur participation sociale et professionnelle;

- et enfin, un principe d'apprentissage tout au long de la vie, qui met l'accent sur des démarches d'apprentissage actives et qui prend en considération les questions de citoyenneté agissante, d'accomplissement personnel et social, de dialogue interculturel, d'emploi (Downes, Nairz-Wirth et Rusinaite, 2017).

Aussi, l'inclusion doit se focaliser non seulement sur les personnes mais aussi sur les systèmes qui les incluent. Malheureusement, on constate que l'inflation du discours sur l'inclusion s'accompagne, à divers degrés selon les pays, d'un maintien ou d'une aggravation des inégalités scolaires, associé à des difficultés d'enseignement pour les professeurs, notamment en début de carrière, ainsi qu'à de possibles résistances familiales. Par le recours croissant de certaines catégories sociales à l'enseignement privé, on assiste à un recul de la mixité socioculturelle à l'école un peu partout dans le monde, aussi bien au Nord qu'au Sud. On peut alors se demander dans quelle mesure les États-nations incarnent l'approche tripartite innovante, structurelle et systémique qui est actuellement recommandée par différentes instances européennes et internationales.

C'est pourquoi l'objectif de ce nouveau dossier de la Revue internationale d'éducation de Sèvres est d'apporter des regards croisés sur la problématique de l'inclusion, en traitant cette question de façon inclusive : il s'agit de prendre en compte, certes, les élèves dits à besoins spécifiques, mais en intégrant leur problématique à celle de l'école en général ainsi qu'à celle de l'évolution de la société moderne vers une société inclusive. L'éducation inclusive a été souvent critiquée pour son manque de ciblage conceptuel. Certains observateurs affirment que le fait d'élargir ce concept pour englober tous les élèves va trop loin, rendant la problématique trop vague (Unesco, 2018). Nous faisons le pari éducatif inverse dans ce numéro, en postulant que l'élargissement de la prise en compte de l'éducabilité de tous les élèves peut être fécond et riche d'enseignements. 
Afin de procéder à une amorce d'état des lieux mondial de la question de l'inclusion, nous avons choisi d'interroger l'évolution des conceptions éducatives au sein de neuf pays : l'Italie, le Brésil, Israël, les États-Unis, la Slovaquie, le Mali, le Laos, la Suisse et la France. Leurs contributions mettent en perspective des évolutions politiques et des réalités nationales complexes, afin de questionner au plan conceptuel et pratique les orientations et d'en opérer une analyse critique néanmoins constructive. Les auteurs se sont en particulier intéressés à toute forme de discrimination qui entrave une pédagogie inclusive, en mettant les obstacles en relation avec des actions susceptibles de promouvoir l'accessibilité et la participation de chacun. En effet, la ségrégation scolaire reste une réalité dans le monde. Ses effets négatifs touchent particulièrement les enfants en situation de handicap, ceux issus de minorités ethniques ou de l'immigration, et à certains degrés les filles. Ce phénomène se trouve lié à plusieurs causalités, lesquelles diffèrent, suivant les pays, en fonction de leur histoire et de leur évolution économique mais renvoient toutes, comme les lecteurs auront l'occasion de le découvrir, à certaines conceptions de l'altérité et à des processus structurels de domination et de discrimination.

Alors que l'enjeu de la suppression des obstacles à l'éducation pour tous les enfants reçoit un soutien quasi universel, les politiques nationales et les pratiques varient selon les régions et les pays. L'inclusion exige que le système évolue pour s'adapter à l'enfant - mais cela se fait rarement, en partie en raison des contraintes de ressources, en partie en raison des mentalités, et en partie en raison d'une interprétation divergente du concept d'inclusion (Unesco, 2018).

Cependant, même si chaque auteur nous montre que le parcours vers l'inclusion est jalonné de multiples obstacles, l'éducation inclusive ne peut être perçue comme un projet utopique qu'au sens étymologique du terme "utopie ": qui n'a pas (encore) de lieu. En ce sens, l'inclusion scolaire constitue un objectif atteignable, qui offre un énorme potentiel pour améliorer la cohésion sociale, les relations interculturelles et les possibilités d'éducation de tous les enfants (Conseil de l'Europe, 2017). Le problème central renvoie donc à la capacité du système à mobiliser tous ses acteurs en faveur de l'inclusion. Autrement dit, l'éducation inclusive exige un changement de paradigme au niveau sociétal. Notre enjeu, en tant que coordinateurs du dossier, est de contribuer modestement à ce changement en proposant aux lecteurs de la Revue une rencontre fructueuse avec les auteurs et avec leur interprétation des situations éducatives qu'ils ont étudiées.

\section{L'INCLUSION : UN PROCESSUS LONG ET JALONNÉ D'ÉTAPES}

Andrea Canevaro développe dans son article le fait que le processus de scolarisation des élèves handicapés dans les classes ordinaires italiennes s'est déroulé en trois temps: un temps d'intégration dans les années soixante-dix, 
avec deux lois qui consacrent le droit de toute personne handicapée à suivre une scolarité normale et sans discrimination, un temps de prise de conscience de la nécessité d'une politique inclusive qui élargisse la notion de besoin éducatif particulier et se détache d'une forme de catégorisation des élèves, un temps de mise en œuvre, depuis les années 1990, de textes réglementaire allant dans ce sens. Selon l'auteur, une école de la diversité nécessite une pédagogie qui humanise, c'est-à-dire qui apprenne à chacun à reconnaître autrui et à appartenir à un groupe. Il s'agit de faire de l'enjeu inclusif une pratique quotidienne, ouverte sur une vision plus large, celle d'une société inclusive, dans une perspective qui lie inclusion et changement social. Au plan pédagogique, cela va au-delà de l'aide apportée à certains élèves par un "enseignant de soutien » (insegnante di sostegno). D'où l'importance de s'extraire, à tous les niveaux, d'actions exclusives (c'est-à-dire adressés à un seul type d'élève) ou unilatérales (c'est-à-dire toujours construites suivant le même schéma opératoire ou conceptuel), pour envisager chaque apprenant comme un être de besoins et diversifier à partir de cela ses postures et gestes professionnels. Il est également important, selon Canevaro, d'élargir le champ de vision scolaire en y incluant les familles, via la construction et le maintien de formes d'alliance avec celles-ci, ainsi qu'au travers de l'aide que l'on peut leur apporter quand elles sont isolées dans leur parcours. L'auteur pointe la nécessité d'une certaine prise de recul et d'une certaine humilité face à la rencontre toujours complexe de parents confrontés à l'inquiétude, qui peuvent sembler inactifs, intrusifs, agressifs, surprotecteurs... L'éducation inclusive passe aussi par le postulat du partenariat avec les familles, qu'il convient de pouvoir envisager à moyen terme comme une ressource, même quand la relation est initialement problématique.

Enicéia Mendes, Mylene Santiago et Katiúscia C. Vargas Antunes introduisent quant à elles les perspectives de l'éducation inclusive au Brésil, en rappelant que l'histoire de ce pays est marquée par de nombreuses inégalités de nature sociale et éducative, notamment en lien avec des facteurs économiques et avec la coprésence de différents groupes culturels. La politique éducative brésilienne, définie depuis 2008 comme étant inclusive, s'est orientée vers la mise en place de formes d'accueil spécialisées qui ne doivent pas se substituer à la scolarisation - ce qui est paradigmatique dans le contexte éducatif brésilien -, et qui sont mises en place, en complément de la scolarisation en classe ordinaire, pour un public cible (les élèves en situation de handicap, ceux qui sont porteurs de troubles du développement et ceux qui présentent un haut potentiel intellectuel). En ce sens, le Brésil se situe actuellement dans l'étape intégrative, nécessaire et fondatrice, de son parcours vers l'inclusion. L'institution de « salles de ressources polyvalentes " (salas de recursos multifuncionais) dans environ un tiers des écoles brésiliennes (recensement de 2011) concrétise en ce sens la volonté du ministère brésilien de l'éducation de mieux répondre aux besoins spécifiques de scolarisation de certains élèves. Selon les auteures, l'articulation entre formation et recherche est au cœur de la capacité des enseignants à se pencher sur leurs 
propres pratiques pour faire face aux problèmes que rencontre le monde scolaire contemporain, et cette articulation passe notamment par un rapprochement à faire entre, d'une part, les professeurs d'université et, d'autre part, les professeurs des écoles et du secondaire. Améliorer le processus d'inclusion scolaire de chaque apprenant exige d'être en mesure de problématiser la façon dont l'école est pensée et construite, afin de surmonter la situation d'exclusion dont souffre un grand nombre d'élèves. Loin de disqualifier les actions intégratives brésiliennes qui garantissent un accès à la scolarisation aux élèves historiquement discriminés, Mendes, Santiago et Vargas Antunes pointent la nécessité d'une approche renouvelée des processus d'apprentissage pour prendre effectivement en compte la diversité des apprenants. En ce sens, un vaste programme de formation continue a été mis en place par le ministère de l'éducation. Il reste à le rendre accessible aux enseignants qui sont éloignés des centres urbains ou qui ne disposent pas d'équipement numérique pour suivre une formation à distance.

\section{L'ÉCOLE INCLUSIVE FACE AUX MULTIPLES FORMES DE DISCRIMINATION}

Zvi Bekerman, prenant appui sur près de cent ans de conflits israélopalestiniens non résolus, fait un état des lieux de l'égalité politique et sociale que l'État d'Israël s'est engagé à assurer, dès sa création, à l'ensemble de ses citoyens, quelle que soit leur religion ou leur appartenance ethnique. Il s'avère que « la discrimination éducative existant à l'égard de la minorité arabo-palestinienne a fait l'objet de nombreuses études ", lesquelles relèvent d'importants écarts en termes d'équipement, de qualification des professionnels de l'éducation, de fréquentation des services d'éducation spéciale, de résultats scolaires. L'auteur pointe la nécessité de développer des rencontres interculturelles (non factices) entre palestiniens et juifs dans le domaine de l'éducation. Il modélise ces rencontres de la façon suivante : celles basées sur la coexistence et les ressemblances interpersonnelles, qui insistent sur l'appartenance commune à l'humanité ; celles animées par l'établissement de projets communs pour transcender l'identité distinctive et défensive de chaque groupe ; celles fondées sur les possibilités de confrontation des points de vue existants, et notamment de ceux issus de la minorité ; et enfin celles axées sur le story-telling, par lesquelles la mise en récit et le partage mutuel des expériences et souffrances vécues permet le développement de l'empathie. L'école inclusive engage ses acteurs à reconsidérer les catégorisations à l'œuvre dans la façon de se représenter autrui, notamment quand des différences d'ordre culturel ou ethnique animent les relations interindividuelles. Quand certains usagers de l'école sont considérés comme étant les représentants d'une minorité, la considération de leur identité par le groupe dit dominant relève généralement d'un processus de construction intellectuelle fondé sur la supposée existence de catégories d'appartenance distinctes (Barry, 2018). 
L'effet délétère en est que l'association de ces catégories relève davantage de la tolérance d'autrui que de la reconnaissance de son altérité. On accepte la présence de l'autre sans chercher à développer la relation avec lui, et cette acceptation a une connotation de condescendance (Jacquart, 1997) : tolérer, c'est accepter (malgré soi) la réalité d'autrui, tandis qu'inclure, c'est s'intéresser à autrui, c'est vouloir une construction mutuelle avec lui.

En passant d'Israël aux États-Unis, on passe de l'identité nationale et religieuse à l'appartenance ethnique, mais la question de l'altérité reste au cœur du propos. La contribution de David J. Connor et Beth A. Ferri met en exergue le fait que la surreprésentation des élèves de couleur dans l'éducation spécialisée est enracinée dans un héritage historique empreint de racisme institutionnel, dont les répercussions sont encore d'actualité. En résumé, suivant les auteurs, les Américains d'origine européenne se sont placés au sommet d'une hiérarchie sociale et raciale, puis ils "ont situé les Afro-Américains au bas de l'échelle, toutes les autres catégories raciales étant classées selon un continuum entre ces deux polarités ». Comme la surreprésentation de certains élèves sur la base de caractéristiques sociales, ethniques, culturelles est représentative de la plupart des pays, il nous apparaît que l'éclairage mené sur la situation aux États-Unis est porteur d'enseignements partout dans le monde. Ce qui est probablement le plus déstabilisant, dans cette contribution, est la permanence des phénomènes de ségrégation, malgré le mouvement des droits civiques, les lois promulguées et la volonté des acteurs éducatifs pour en finir avec la surreprésentation. Connor et Ferri mettent en évidence l'apport de la recherche et de l'intersectionnalité pour nous aider à comprendre et agir sur le phénomène. Comme nous le montrent ces auteurs, le droit d'autrui à être culturellement différent de soi est un enjeu fondamental de l'école inclusive, non encore réalisé. Le défi est non seulement ici de repenser le rapport à l'autre, mais également de découvrir en quoi autrui permet de transformer ce que l'on pense savoir de soi.

René Lužica et Arnaud Segretain pointent, dans leur article, l'existence d'une stratification sociale de la société slovaque qui a eu en particulier pour conséquence le fait que la majorité des Roms forme une communauté marginalisée vivant dans des espaces circonscrits et isolés en zones urbaines ou rurales. De plus, bien que des matières telles que la langue rom, la culture rom, l'histoire des Roms aient été officiellement incluses dans les programmes de formation continue du personnel enseignant des écoles primaires, la concrétisation d'un environnement scolaire inclusif reste un processus difficile pour cette population d'élèves, notamment en raison d'un manque de professeurs compétents et volontaires pour enseigner ces matières. Une étude du parcours éducatif des élèves roms, menée en 2013, a montré qu'un nombre minime d'entre eux était satisfait de sa vie actuelle, et que les relations à l'école étaient compliquées en raison d'obstacles à la communication et de comportements ségrégatifs. Aussi, selon les auteurs, l'école reste pour les Roms un environnement étranger et utilisant une langue étrangère. Leur contribution montre, en écho aux travaux de 
Goffman (1975), que les processus d'attribution d'étiquettes sociales et culturelles sont susceptibles de produire des formes de stigmatisation. L' « étiquetage » est en effet producteur de différenciations, de mises à distance, en même temps qu'il marque un refus (affiché ou inavoué) de coexister, de partager un espace social. L'école slovaque, sur son chemin vers l'inclusion, apprend actuellement à être multiple, mais n'est pas encore diverse. Autrement dit, le "pluri » n'est pas l'«inter»: une approche éducative fondée sur l'identification première de besoins universellement partagés n'est pas équivalente à une conception basée sur l'identification de besoins vus comme étant spécifiques, particuliers (Barry, 2018). Il s'agirait ici de créer du lien, des influences réciproques, au-delà d'une coprésence et d'une (pseudo) co-acceptation.

\section{L'INCLUSION SCOLAIRE ET LA QUESTION DU GENRE}

Les contributions dédiées au Mali et au Laos explorent les conséquences des discriminations liées à la mixité sur l'inclusion de certains groupes dans les processus éducatifs, la discrimination constituant le fait de traiter différemment certaines personnes par rapport à d'autres.

Dans son article, Seydou Loua montre qu'au Mali, les discriminations frappant les femmes perdurent de l'éducation de base à l'université. Selon l'auteur, "si des améliorations ont été enregistrées dans certains aspects de l'éducation des filles grâce à l'attention que celle-ci suscite dans le monde, il reste beaucoup à faire pour atteindre la parité des genres " dans l'accès à l'école, le maintien dans le système scolaire, l'orientation et la qualité des enseignements reçus. Les obstacles à la parité analysés par Seydou Loua sont multiples, complexes et difficiles à dépasser, pour certains. En particulier, les obstacles liés aux traditions et aux croyances religieuses nécessitent non seulement des moyens mais aussi des actions durables pour influencer les mentalités, les représentations sociales et les conceptions du statut de la femme. En effet, comme les actions discriminatoires se fondent sur des distinctions, elles sont liées à des préjugés, c'est-à-dire à des jugements qui précèdent la connaissance (Bertrand, 2018). Les discriminations présentent donc le paradoxe d'être issus d'une situation de méconnaissance ou d'ignorance et de déterminer le comportement et les choix des personnes qui portent certains préjugés. Aussi, s'agissant de l'éducation mixte au Mali, une coalition des acteurs étatiques, internationaux et locaux est requise, de façon à, dans un premier temps, mettre au jour les préjugés puis, dans un second temps, faire construire de nouvelles connaissances et fournir des moyens matériels pour les faire évoluer. Dans un pays à faible revenu, se battant encore pour élargir l'accès au système éducatif, penser l'éducation en termes d'enjeux inclusifs peut apparaître comme étant un luxe car d'autres nécessités se manifestent fortement, comme la construction des écoles et des classes ou la formation des enseignants. Or l'ampleur des discriminations peut annihiler tout effort pour améliorer la qualité de l'éducation. 
Dans sa contribution sur le Laos, Kongsy Chounlamany explore la disparité des opportunités éducatives selon le genre et l'ethnicité, disparité qui se manifeste dans le fait que, parmi le nombre important d'élèves qui décrochent scolairement, figure une majorité de filles et d'enfants issus de minorités ethniques. D'après l'auteure, «le fossé existant entre les sexes et l'importance du pouvoir masculin sont également clairement visibles dans les différences de statut social au sein de l'organisation étatique ». L'exclusion la plus manifeste des opportunités éducatives touche au Laos les filles appartenant aux minorités ethniques. Animés par une volonté de développement économique et une politique d'attractivité des investissements internationaux, les pouvoirs publics ont tenté de réduire les discriminations frappant les filles et les minorités ethniques dans le secteur de l'éducation. Néanmoins, les résultats tardent à venir car des difficultés structurelles entravent les projets éducatifs. Par exemple, l'éloignement des communautés rurales et la pauvreté extrême rendent difficile le développement de l'offre éducative et l'affectation des enseignants sur certains postes. De surcroît, dans un contexte de migrations internes et externes fortes, il est difficile de prévoir des mesures éducatives pérennes et ciblant les plus vulnérables. Le propos de Chounlamany montre la nécessité de faire de l'équité et de la justice sociale un projet institutionnel continu (Unesco, 2009), non seulement en identifiant les inégalités et les formes de discrimination, mais également en interrogeant les processus et réalités qui génèrent celles-ci. Il s’agit de mettre en œuvre des conditions d'effectivité du droit à l'éducation, comme la dotation en ressources, l'accessibilité des écoles, l'acceptabilité sociale du public éduqué et l'adaptabilité des contenus et des pratiques, ces indicateurs permettant d'évaluer la réalité d'un projet inclusif dans un système éducatif national (Potvin, 2014).

\section{LA NÉCESSITÉ D'UNE FORMATION À LA RÉFLEXIVITÉ ET À L'INTERSUBJECTIVITÉ}

La mobilisation de tous les acteurs pour l'éducation inclusive mentionnée précédemment ne peut se concrétiser sans une transformation de la formation et du travail enseignant, comme l'illustrent les contributions consacrées à la France et à la Suisse.

Corinne Monney analyse les liens ambigus entre les prescriptions d'inclusion, la formation des enseignants et les dispositifs d'éducation inclusive, dans un contexte suisse marqué par la décentralisation de l'éducation et des changements législatifs importants. En réponse aux prescriptions sociopolitiques nationales et internationales, l'école inclusive en Suisse romande " met en perspective des postulats régulateurs contradictoires : d'un côté, des signalements d'élèves, des dépistages, des orientations, des sélections de plus en plus précoces »; de l'autre côté, "une poussée prescriptive de l'école normative vers une école intégrative et inclusive ». Les enseignants représentent évidemment des acteurs 
clefs face à ces postulats contradictoires. La contribution de Monney montre que la mise en œuvre d'une école inclusive en Suisse porte ainsi moins sur la prescription de droits (qui est pléthorique) que sur sa nécessaire « renormalisation collective ", dans les faits. En effet, comme le dit l'auteure, "le prescrit structure les rapports en formation et donne un cadre aux dispositifs d'éducation inclusive. Mais, ce faisant, il laisse rémanentes des couches successives de principes de justice contradictoires qu'il se contente de faire valoir à ce stade par une fonction rhétorique ». L'étude ainsi menée dans le canton de Vaud confirme en ce sens les conclusions d'une recherche précédente réalisée dans le canton de Genève (Soussi et al., 2012) : alors que la nécessité d'une transformation curriculaire se trouve au centre des discours prescriptifs et des politiques de lutte contre les inégalités scolaires, cette nécessité est peu instrumentée par les acteurs de l'éducation et de la formation, et elle l'est souvent maladroitement. En effet, si l'inclusion se limite dans les faits à la coprésence en classe d'élèves aux profils diversifiés, sans qu'une réflexion de fond ne soit menée par l'équipe éducative sur l'accessibilité sociale et intellectuelle de chacun d'eux, elle risque de n'être qu'une "subtilité sémantique " (Gillig, 2006), permettant de "se donner bonne conscience » tout en ayant le sentiment d'un échec pédagogique.

Ce constat, centré sur les gestes professionnels, va dans le sens de celui que fait Alexandre Ployé en France, quand il explore au plan intersubjectif la relation entre les professeurs et les élèves en situation de handicap. En effet, si à l'échelle macro le projet inclusif repose sur une approche anthropologique féconde de la personne handicapée, laquelle est envisagée suivant des principes d'accessibilité et de compensation, à l'échelle micro, l'agir enseignant est néanmoins difficilement pensé en termes d'adaptations et de différenciation pédagogique. Il n'est pas aisé de faire en permanence preuve d'attention, d'ajustements réguliers et de créativité, mais ce n'est pas tant, selon l'auteur, un défaut de maîtrise didactique ou d'organisation qui limite les pratiques d'inclusion, mais plutôt l'émergence d' "éprouvés psychiques spécifiques attachés à la rencontre dans la classe avec un élève handicapé ». Ployé montre que la réussite de l'école inclusive repose sur la capacité plus ou moins grande des enseignants à « intégrer psychiquement le sentiment d'inquiétante étrangeté » que provoque la rencontre avec un autrui parfois vécu comme étant radicalement différent de soi. De plus, la crainte de ne pas être à la hauteur du postulat inclusif, surtout quand on adhère à celui-ci, peut provoquer des moments de découragement professionnel, et donc des formes de limitation de l'agir enseignant. Aussi, il est fondamental de prendre en compte, en formation initiale comme en formation continue, «les conditions d'une vulnérabilisation identitaire des enseignants ", en écho à la vulnérabilité de certains élèves. Cela passe par une réflexivité qui se situe tout autant sur le plan de l'éthique que sur celui des actions professionnelles, qui soit "incarnée et non subie », et qui permette aux professeurs de s'extraire d'une éventuelle approche techniciste de l'enseignement/apprentissage. En résumé, la réussite de la scolarité des élèves passe par une transformation des postures 
pédagogiques et nécessite en formation des dispositifs donnant accès à la subjectivité des acteurs de l'éducation.

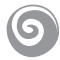

Comme les lecteurs auront l'occasion de le découvrir dans ce numéro de la Revue, le défi de l'école inclusive marque un changement de paradigme qui se réalise différemment dans les pays dont la situation est ici analysée. Mais l'on peut identifier certains points communs entre les contributions, comme la nécessité d'une approche systémique de l'éducation, du passage d'une perspective pluriculturelle à une perspective interculturelle quand il est question d'ethnicité, et surtout d'une réflexion éthique sur la diversité humaine et les notions d'égalité et d'équité. Pour approfondir cette question, les lecteurs pourront se référer aux références bibliographiques proposées par Bernadette Plumelle, lesquelles retracent la chronologie de l'élaboration du concept d'éducation inclusive par les organisations internationales, questionnent le concept d'inclusion et ses récentes évolutions, et témoignent de stratégies et pratiques relevant de différentes aires géographiques, en s’intéressant notamment à la scolarisation des élèves en situation de handicap ou discriminés en raison de leur origine ethnique et sociale.

\section{BIBLIOGRAPHIE}

BARRY V. (2018) : "L'école inclusive au prisme de l'altérité ", La nouvelle revue. Éducation et société inclusives, $\mathrm{n}^{\circ}$ 82, p. 9-25.

BERTRAND N. (2018) : "Transformation des représentations sur le handicap chez des élèves de cycle 3 : l'impact de la mise en situation ", La Nouvelle Revue Éducation et société inclusives, $\mathrm{n}^{\circ}$ 82, p. 79-94.

CONSEIL DE L'EUROPE (2017) : Lutter contre la ségrégation scolaire en Europe par l'éducation inclusive, document de synthèse, Strasbourg : Conseil de l'Europe.

DOWNES P., NAIRZ-WIRTH E., RUSINAITE V. (2017) : «Structural Indicators for Inclusive Systems in and around Schools », NESET II report, Luxembourg: Publications Office of the European Union. DOI : 10.2766/200506

GILLIG J.-M. (2006) : «L'illusion inclusive ou le paradigme artificiel », La Nouvelle Revue de l'Adaptation et de la Scolarisation, $\mathrm{n}^{\circ}$ 36, p. 119-125.

GOFFMAN E. (1975) : Stigmates. Les usages sociaux des handicaps, Paris : Éditions de Minuit.

JACQUARD A. (1997): Petite philosophie à l'usage des non-philosophes, Paris: Calmann-Lévy.

POTVIN M. (2014) : « Diversité ethnique et éducation inclusive : fondements et perspectives ", Éducation et sociétés, n 33, p. 185-202.

SOUSSI A., NIDEGGER C., DUTREVIS M. et CRAHAY M. (2012) : "Un réseau d'enseignement prioritaire dans le canton de Genève : quels effets sur les élèves ?", Revue française de pédagogie, ${ }^{\circ}$ 178, p. 5-12.

UNESCO (2018) : Rapport mondial de suivi sur l'éducation 2020. Inclusion et éducation. Note conceptuelle, Paris : Unesco. 